\title{
Dispensação Farmacêutica: proposta de um modelo para a prática
}

\author{
Dispensation of Pharmaceuticals: \\ proposal of a model for dispensation
}

Daniela Angonesi ${ }^{1}$

Marcela Unes Pereira Rennó ${ }^{1}$

${ }^{1}$ Faculdade de Ciências Biológicas e da Saúde, Centro Universitário Newton Paiva. Av. Silva Lobo 1731, Nova Granada. 30575-100 Belo Horizonte MG.

Abstract The dispensation of pharmaceuticals is an activity that cannot be restricted only to the delivery of medication. The pharmacist must ensure various conditions to ensure that the patient uses the medication in the best possible manner. As it is a professional activity and has been established for pharmaceutical care, dispensation should also have a philosophy of practice, a process of care, and a system for managing the practice. This paper proposes a new definition of dispensation and describes its central elements, considering the reality of established pharmacists, so that it can be effectively implemented.

Key words Dispensation, Pharmaceutical care, Correct use of medication, Community pharmacy
Resumo A dispensação é uma atividade farmacêutica que não pode se restringir apenas à entrega do medicamento. O farmacêutico deve promover as condições para que o paciente faça uso do medicamento da melhor maneira possível. Como se trata de uma atividade profissional, e assim como foi estabelecido para a atenção farmacêutica, a dispensação também deve possuir uma filosofia de prática, um processo de cuidado e um sistema de gestão da prática. Este trabalho propõe uma nova definição de dispensação e descreve os seus elementos centrais considerando a realidade dos estabelecimentos farmacêuticos de forma que possa efetivamente ser implementada.

Palavras-chave Dispensação, Atenção farmacêutica, Uso correto de medicamento, Farmácia comunitária

daniangonesi@hotmail.com 


\section{Introdução}

Nos últimos quinze anos a prática farmacêutica tem passado por uma transformação chamada por muitos de revolucionária. Desde o trabalho de Hepler e Strand ${ }^{1}$, onde estabeleceram que a prática farmacêutica deve possuir uma filosofia apropriada e uma estrutura organizada dentro da qual se exerça essa prática, propondo o conceito de Atenção Farmacêutica, muito se têm discutido e realizado no âmbito da profissão farmacêutica.

O foco do trabalho do farmacêutico, especialmente daqueles que trabalham em farmácias comunitárias, passa a ser o paciente. Todas as suas ações e responsabilidades quando centradas no paciente usuário do medicamento trazem benefícios diretos para ele e para o sistema de saúde.

Especialmente no Brasil, essa transformação paradigmática tem contribuído de forma significativa para a evolução da profissão e criado expectativas nos profissionais em relação à dignificação dessa atividade, oferecendo uma oportunidade de resgate da relação farmacêutico-paciente há muito tempo perdida nas farmácias comunitárias.

A evolução da profissão no Brasil sofreu grande influência de interesses comerciais, tendo contribuído para o abandono da farmácia por parte do profissional e para a perda da relação com o paciente. A promoção da Atenção Farmacêutica, especialmente da sua filosofia, envolvendo todas as atividades dos farmacêuticos relacionadas ao paciente pode ser uma forma de recuperar sua função nas farmácias comunitárias².

No Brasil, o conceito de Atenção Farmacêutica que tem sido aceito é aquele constante na Proposta de Consenso Brasileiro de Atenção Farmacêutica:

É um modelo de prática farmacêutica, desenvolvida no contexto da Assistência Farmacêutica. Compreende atitudes, valores éticos, comportamentos, habilidades, compromissos e corresponsabilidades na prevenção de doenças, promoção e recuperação da saúde, de forma integrada à equipe de saúde. É a interação direta do farmacêutico com o usuário, visando uma farmacoterapia racional e a obtenção de resultados definidos e mensuráveis, voltados para a melhoria da qualidade de vida. Esta interação também deve envolver as concepções dos seus sujeitos, respeitadas as suas especificidades bio-psico-sociais, sob a ótica da integralidade das ações de saúde $e^{3}$.

Nesta proposta, todas as atividades farmacêuticas relacionadas ao paciente, como a indica- ção de medicamentos que não necessitam de prescrição médica, a dispensação e a orientação farmacêutica, são consideradas atividades componentes da prática da atenção farmacêutica juntamente com o acompanhamento farmacoterapêutico. Por isso, e ao englobar as ações de saúde concordantes com os princípios do SUS esta é uma proposta que considera a realidade brasileira da profissão farmacêutica e das farmácias comunitárias no país².

Considerando a missão da prática farmacêutica definida pela Organização Mundial de Saú$\mathrm{de}^{4}$, as atividades de educação em saúde, especialmente relacionadas ao uso correto de medicamentos, a dispensação e a indicação farmacêutica podem interferir de maneira significativa no uso adequado de medicamentos pelas pessoas e pela sociedade.

A dispensação, sendo uma das atividades da prática farmacêutica, deveria seguir os princípios preconizados pela OMS na Declaração de Tóquio ${ }^{4}$. Ou seja, além de entregar o medicamento ou produto para saúde, o farmacêutico deve promover as condições para que o paciente use-o da melhor maneira possível. Promover as condições para o uso adequado não é apenas fornecer algumas informações no momento da entrega do medicamento.

A dispensação ideal deve aliar o caráter técnico do procedimento de entrega que garanta o recebimento de um medicamento ou dispositivo dentro dos padrões de qualidade, segurança e orientações que promovam o uso adequado e apropriado dos medicamentos.

Mas como isso pode ser realizado na prática? Essa pergunta deve ser respondida primeiramente criando-se um modelo que contemple os objetivos da dispensação, entendida como uma atividade relacionada à atenção farmacêutica, mas diferente dela, nos seus aspectos práticos. Na dispensação, o farmacêutico atende o paciente no balcão, ou às vezes numa sala privativa, mas não tem tempo e nem todas as informações para uma avaliação completa como a que ocorre no acompanhamento farmacoterapêutico. Portanto, é necessário redefinir a dispensação e propor os processos envolvidos segundo os seus objetivos e considerando a realidade dos estabelecimentos farmacêuticos.

Como se trata de uma atividade profissional, e assim como foi estabelecido para a atenção farmacêutica por Cipolle et al. ${ }^{5}$, a dispensação também deve possuir uma filosofia de prática, um processo de cuidado e um sistema de gestão da prática. 
O objetivo deste trabalho é propor um novo conceito e um modelo de dispensação baseado em um fluxograma que orienta as ações principais que devem ser realizadas pelo farmacêutico durante a entrevista e orientação do paciente que procura uma farmácia para adquirir um medicamento com prescrição médica. Esta proposta foi feita com base em revisão da literatura com ênfase no atual paradigma da atenção farmacêutica. Foram os pilares da prática da atenção farmacêutica proposta por Cipolle et al. ${ }^{5}$ e a experiência das autoras que nortearam a elaboração deste modelo de prática.

\section{Definição}

Embora existam tentativas de consenso de termos e definições relacionadas à prática farmacêutica, incluindo a dispensação, ainda existem muitas contradições na legislação e diferentes propostas de conceitos. Na prática, a dispensação continua sendo tratada como um ato de entrega de um produto desprovido de sua função técnica e profissional.

Uma análise dos documentos oficiais sobre dispensação nos mostra que não há uma definição de dispensação que poderia ser considerada oficial $^{6}$. Há pelo menos três definições diferentes para a dispensação. Embora haja um avanço demonstrado pelo Ministério da Saúde através da Política Nacional de Medicamentos - $\mathrm{PNM}^{7}$ em que o aspecto comercial da atividade é excluído, dando-lhe um caráter profissional deixando claro que o farmacêutico é responsável não só pelo fornecimento do medicamento, como também pela orientação para o seu uso adequado e inserindo a atividade num grupo multiprofissional de assistência à saúde (a assistência farmacêutica), não houve mudanças nos conceitos utilizados pelos principais órgãos reguladores sanitário e profissional, respectivamente a Agência Nacional de Vigilância Sanitária - ANVISA e o Conselho Federal de Farmácia - CFF. Estes últimos repetem a definição da Lei no 5991, de 17 de dezembro de 1973: ato de fornecimento ao consumidor de drogas, medicamentos, insumos farmacêuticos e correlatos, a título remunerado ou $n \tilde{a} o^{8}$, incluindo a palavra orientação.

Na Espanha, o Consenso de Atenção Farmacêutica, inclui a dispensação como uma das atividades da Atenção Farmacêutica relacionandoa com as outras voltadas ao paciente. Esse documento descreve que a dispensação é um ato profissional complexo que não deve ser considerada uma atividade final e sim como um suporte para realizar a atenção farmacêutica. Destaca-se que farmacêutico deve ter uma atitude ativa ao entregar o medicamento e/ou produto sanitário em condições ótimas e de acordo com as normas legais vigentes, protegendo o paciente da possível aparição de problemas relacionados com os medicamentos ${ }^{9}$.

Dupim $^{10}$ afirma que a dispensação é um procedimento que tanto pode representar a etapa final que sintetiza todas as anteriores, como pode ser o ponto de partida para o encaminhamento do paciente a outros serviços de saúde. Segundo esse autor, no momento da dispensação o farmacêutico ouve o usuário, esclarece suas dúvidas e complementa as informações fornecidas por outros profissionais de saúde sobre o uso e a guarda do medicamento, com o objetivo de evitar o aparecimento de problemas que possam comprometer a terapêutica.

A definição de dispensação apresentada por Marin et al. ${ }^{11}$ é o proposto por Arias ${ }^{12}$ :

A dispensação é o ato farmacêutico de distribuir um ou mais medicamentos a um paciente, geralmente como resposta à apresentação de uma prescrição elaborada por um profissional autorizado. Neste ato, o farmacêutico informa e orienta o paciente sobre o uso adequado do medicamento. São elementos importantes dessa orientação, entre outros, a ênfase no cumprimento do regime de dosificação, a influência dos alimentos, a interação com outros medicamentos, o reconhecimento de reações adversas potenciais e as condições de conservação do produto.

Diante dessas definições, cabe perguntar: por que, geralmente, a dispensação não é realizada das maneiras apresentadas? Um dos motivos pode ser a falta de procedimentos e recomendações para colocar em prática o que de fato entende-se por dispensação de medicamentos.

Não há como desvincular o caráter comercial dos estabelecimentos farmacêuticos atualmente, pois essa realidade decorre de um processo cultural e envolve questões de ordem sócio-econômicas amplas. Então se deve entender a dispensação como uma prática que seja eficiente e ágil para abranger as dimensões técnicas e comerciais.

Segundo o Consenso Espanhol ${ }^{9}$ a dispensação deve servir como:

- fonte de informação para os pacientes sobre o medicamento que irá utilizar;

- filtro para detecção de situações nas quais haja um risco de ocorrência de problemas relacionados a medicamentos;

- fonte de informação ao farmacêutico, a partir da qual ele tome a decisão mais benéfica para 
o paciente, que pode ser umas das seguintes: dispensar o medicamento segundo a prescrição; oferecer uma assistência complementar mediante outro serviço de atenção farmacêutica (educação sanitária, farmacovigilância, seguimento do tratamento farmacológico); ou não dispensar sem remissão ou prévia consulta ao médico ou dentista.

Além disso, descreve que a dispensação deve cumprir três requisitos básicos: atender a $100 \%$ dos consumidores, ser ágil e eficiente e estar integrada à rotina diária do profissional. A dispensação deve ser realizada pelo farmacêutico ou sob sua supervisão.

A agilidade e a eficiência exigem que as informações fornecidas sejam as mínimas necessárias para uma farmacoterapia adequada. Isso significa que não deve ser condição na dispensação o fornecimento de informações sobre interações medicamentosas e reações adversas bem como a avaliação de todas as necessidades farmacoterapêuticas do paciente, já que nessa prática não se dispõe de tempo para obtenção de informações mais detalhadas. Por outro lado, devem-se identificar as situações que possam comprometer o tratamento, indicando a necessidade de encaminhar o paciente para um atendimento mais completo e resolver problemas possíveis no balcão.

Vale destacar que além do fornecimento de informações é importante promover o desenvolvimento de habilidades e competências para que o paciente manifeste atitude positiva em relação à sua farmacoterapia, assumindo comportamentos que representem o uso correto/adequado dos medicamentos.

Para atender $100 \%$ da população a dispensação deverá ser realizada com a ajuda dos auxiliares sob responsabilidade do farmacêutico. Para tanto é necessário que os auxiliares sejam capacitados para realizar esse serviço e identificar as situações nas quais somente o farmacêutico pode tomar decisão.

E para estar integrada à sua rotina diária, o profissional deve organizar suas atividades para que possa priorizar a dispensação. Para isso é necessário que haja uma mudança do farmacêutico em direção ao novo paradigma de cuidado ao paciente e, desse modo, perceber a dispensação como a atividade mais importante dentro das farmácias comunitárias.

Assim, o conceito de dispensação de medicamentos proposto é: prática farmacêutica na qual o profissional fornece medicamentos se responsabilizando por educar o paciente sobre o uso adequado dos mesmos e pela triagem de possí- veis problemas relacionados à farmacoterapia que necessitem de cuidado em outros serviços farmacêuticos.

\section{Filosofia}

Na proposição da Atenção Farmacêutica, Hepler e Strand ${ }^{1}$ destacaram a necessidade de mudança de paradigma profissional, ou seja, um novo jeito de entender a profissão. Da mesma forma, no nosso entendimento, o sucesso da dispensação depende dessa mesma mudança. O farmacêutico deve assumir nas farmácias uma atitude de cuidador e não de vendedor.

Assim, baseado no proposto para a atenção farmacêutica e considerando as peculiaridades da dispensação, a filosofia dessa prática deve ser sustentada pelos seguintes pilares:

- atender à necessidade social de reduzir a morbimortalidade de medicamentos;

- estabelecer uma relação de confiança entre o farmacêutico e o paciente;

- atender o paciente de maneira individualizada e com foco nas suas necessidades relacionadas ao uso adequado do medicamento;

- responsabilizar-se por educar o paciente para o uso adequado do medicamento e identificar as situações que necessitem de outros cuidados.

\section{Processo de cuidado}

O objetivo do processo de cuidado do paciente é direcionar a conduta do farmacêutico durante a dispensação. Neste momento o farmacêutico obtém informações, analisa e toma decisões em relação à orientação sobre o uso adequado do medicamento e à identificação de possíveis problemas que necessitem de encaminhamento para outros serviços. O processo de cuidado na dispensação apresentado está no formato de fluxograma (Figura 1) e pretende estabelecer um procedimento padrão a ser adotado em qualquer estabelecimento que realize a dispensação direta ao usuário. Isso proporciona uma identidade profissional da prática e consequente reconhecimento social do farmacêutico. O fluxograma orienta os passos necessários para obter informações que o auxiliem na tomada de decisões, que é individual. Porém, o farmacêutico deve se sentir livre para utilizar outros questionamentos que julgar necessários para atender as necessidades do paciente na dispensação.

Inicialmente o farmacêutico deve avaliar se há prescrição, pois isso indica que o paciente passou por uma avaliação médica/odontológica pré- 


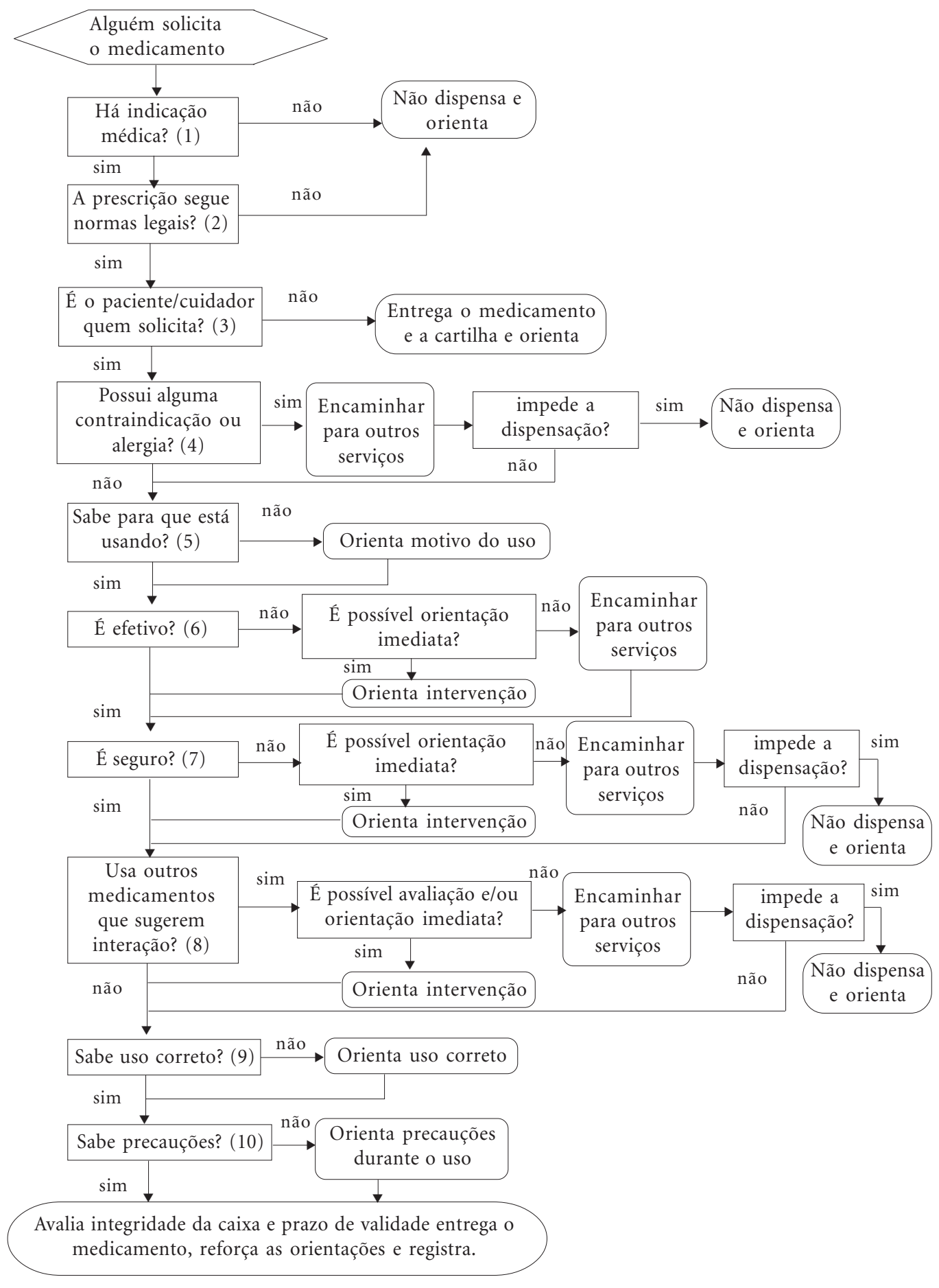

Figura 1. Fluxograma de ações durante a dispensação de um medicamento

via, na qual foi estabelecido o diagnóstico que justifique o uso do medicamento (pergunta 1). Caso não haja prescrição considerar a possibilidade do paciente relatar ocorrência de indicação médica ou odontológica prévia mesmo não apresentando a prescrição. Essa é uma prática comum, principalmente para aquisição de medicamentos de uso crônico que necessitam da pres- 
crição, devido a vários fatores que resultam na desvalorização da importância deste documento por parte dos pacientes, prescritores e farmacêuticos. Nesse caso, o farmacêutico deve avaliar se dispensa o medicamento considerando dois aspectos: não entregar o medicamento pode causar o descontrole da doença ou entregar um medicamento que não foi indicado pelo profissional habilitado pode causar prejuízos à saúde do paciente. É fundamental o farmacêutico orientar este sobre a importância da apresentação da prescrição para minimizar a ocorrência desses problemas e em longo prazo modificar o comportamento das pessoas.

$\mathrm{Na}$ presença da prescrição deve-se avaliar o cumprimento das exigências legais (pergunta 2). A Lei n. $5.991 / 73^{8}$ que dispõe sobre o controle sanitário do comércio de drogas, medicamentos, insumos farmacêuticos e correlatos estabelece que somente deve ser aviada a receita que estiver escrita a tinta, em vernáculo, por extenso e de modo legível, observados a nomenclatura e o sistema de pesos e medidas oficiais; que contiver o nome e o endereço residencial do paciente e, expressamente, o modo de usar o medicamento; e ainda que contiver a data e a assinatura do profissional, endereço do consultório ou da residência e o número de inscrição no respectivo Conselho profissional ${ }^{8}$ Já o aviamento de receitas contendo medicamentos sob controle especial deve obedecer às disposições da Portaria n. 344/98 ${ }^{13}$.

Embora esses critérios sejam exigidos por lei, na prática nem sempre as prescrições estão completas. Mas alguns itens são essenciais para garantir a segurança da dispensação: o nome, o registro no Conselho Profissional e a assinatura do prescritor e, ainda de forma legível, o nome do medicamento, a concentração, a dose, o intervalo de administração, a quantidade e a duração do tratamento. Essas informações são fundamentais para que o farmacêutico possa orientar a forma de uso correto do medicamento.

O farmacêutico deve entrar em contato com o profissional prescritor para esclarecer eventuais problemas ou dúvidas que tenha detectado no momento da avaliação da receita. Se julgar necessário, o farmacêutico não deve realizar a dispensação e encaminhar o paciente de volta ao médico para correção ou complementação da prescrição. Nas situações em que a prescrição está completa continua o procedimento de dispensação proposto.

Se a solicitação for feita por uma pessoa que não seja o usuário ou o cuidador (pergunta 3), o comprador deve ser orientado quanto ao modo de uso correto do medicamento e receber uma cartilha com informações sobre o medicamento, se a tiver disponível. A cartilha é importante para oferecer as informações básicas sobre o uso de medicamentos já que o comprador geralmente não tem condições de fornecer os dados sobre a saúde do paciente, necessários para a dispensação, como está sendo proposta. O farmacêutico também informa e orienta o comprador sobre sua necessidade de avaliar o paciente, e se coloca à disposição para o esclarecimento das dúvidas deste sobre a utilização do medicamento. Se for o paciente ou o cuidador quem solicita o medicamento a dispensação é feita de acordo com o fluxograma.

A avaliação de contraindicação ou alergia (pergunta 4) deve ser feita com todos os pacientes, independente de ser a primeira vez que vai utilizar o medicamento ou não. Deve-se dar atenção especial aos casos de gravidez e de lactação e naqueles em que o paciente faça referência clara de doenças que sugiram de contraindicação (por exemplo, asma e betabloqueador), já que é impossível avaliar todas as possibilidades de contraindicação dos medicamentos no balcão da farmácia. Nos casos de uso inicial do medicamento, deve-se perguntar sobre a utilização prévia deste e de similares a fim de investigar a possibilidade de alergia. Se for identificada uma contraindicação ou alergia, o paciente deve ser encaminhado ao médico para confirmação e ajustes na terapêutica. O farmacêutico deve avaliar se dispensa o medicamento levando em consideração a situação atual do paciente pois o risco da não dispensação pode ser maior do que o da possível contraindicação.

O conhecimento do motivo do uso (pergunta 5) é importante para a adesão do paciente ao tratamento, já que ele pode perceber a importância da utilização do medicamento para o controle da sua doença e as consequências de não usar. Se o paciente não sabe informar qual o diagnóstico, o farmacêutico deve realizar outros questionamentos para tentar identificar o possível motivo do uso considerando que alguns medicamentos possuem várias indicações. Nos casos em que o paciente relatar que sabe o motivo do uso é importante verificar se o conhecimento demonstrado é satisfatório. Não é necessário fazer essa pergunta toda vez que o paciente for adquirir o medicamento, pois, espera-se que após a primeira orientação o paciente já possua o conhecimento do motivo do uso.

Para avaliar a efetividade (pergunta 6), devese realizar perguntas adequadas à doença que o 
paciente apresenta. Por exemplo, se for hipertensão, pergunta-se sobre os valores da pressão arterial. Os pacientes que não fazem monitoramento do tratamento devem ser orientados sobre a importância desses procedimentos. Caso o medicamento não esteja sendo efetivo, tentar avaliar a causa, especialmente o uso correto e as interações. No caso do uso incorreto pode-se intervir imediatamente para resolver o problema. Algumas interações também podem ser resolvidas no balcão. Essas situações serão abordadas no decorrer do fluxograma. Se não for possível avaliar a causa, ou o problema não puder ser resolvido neste momento, o paciente deve ser encaminhado a outros serviços farmacêuticos (acompanhamento farmacoterapêutico) ou diretamente ao médico com um breve relato da avaliação farmacêutica e, de preferência, por escrito. Se o paciente ainda não iniciou o tratamento não será possível avaliar a efetividade, mas é importante orientar quais são os procedimentos para verificar o resultado, incluindo o tempo necessário para observar o efeito esperado do medicamento.

Em relação à segurança (pergunta 7) deve-se realizar perguntas sobre sintomas que incomodem o paciente e avaliar se a queixa pode estar relacionada ao medicamento. Caso haja suspeita da relação entre as queixas e o medicamento, tentar avaliar a causa, especialmente o uso correto e as interações. Assim como na avaliação de efetividade, na presença do uso incorreto pode-se intervir imediatamente para resolver o problema, e no caso das interações, algumas podem ser resolvidas durante a dispensação. Se não for possível avaliar a causa, ou o problema não puder ser resolvido neste momento, o paciente deve ser encaminhado a outros serviços farmacêuticos ou diretamente ao médico com um breve relato da avaliação farmacêutica e, de preferência, por escrito. Além disso, deve-se avaliar se o medicamento pode ser entregue, considerando a relação risco-benefício da situação apresentada. Se o paciente ainda não iniciou o tratamento também não será possível avaliar a ocorrência de reações adversas, mas em alguns casos pode-se orientar sobre alguns sintomas que sugerem reações não desejadas.

A avaliação do uso de outros medicamentos (pergunta 8) tem o objetivo de identificar a presença de interações que possam reduzir a efetividade e a segurança do tratamento. Essa avaliação depende da qualidade da informação fornecida pelo paciente, que pode não relatar todos os medicamentos em uso, e do conhecimento e experiência do farmacêutico. Feita a identificação da interação, o farmacêutico deve avaliar se é possível uma intervenção imediata (alterar horário, suspender medicamento de venda livre) ou se será necessário encaminhar para o prescritor. Se não for possível realizar a avaliação no momento, pode-se adotar o critério de encaminhar o paciente que utiliza mais de três medicamentos. Nos casos em que se identificou uma importante interação, deve-se avaliar se o medicamento deve ser entregue considerando a relação risco-benefício da situação apresentada.

É fundamental que todos os pacientes e cuidadores conheçam e sejam estimulados a realizar a forma correta de administração dos medicamentos de forma a contribuir para a efetividade e a segurança do tratamento (pergunta 9). Considera-se uso correto a utilização da dose, a via e os intervalo prescritos, com técnica de administração adequada e pelo tempo estipulado. O farmacêutico deve se certificar de que o paciente realmente sabe o modo de administração, não se restringindo a pergunta: Você sabe como utilizar o medicamento?. É importante verificar qual a forma que o paciente usa ou pretende utilizar o medicamento e, a partir disso, avaliar se está correto. Caso seja constatado que o paciente não tem o conhecimento correto, deve-se realizar a orientação e nos retornos confirmar se o paciente está fazendo o uso exato.

As precauções durante o uso (pergunta 10) são as recomendações para o armazenamento adequado, a prevenção de reações adversas e as atitudes que aumentam a comodidade do tratamento. Como exemplo, a utilização de protetor solar no caso de medicamentos que causem fotossensibilidade e o aumento da ingestão de certos nutrientes que podem estar diminuídos devido ao medicamento. Da mesma forma, o farmacêutico deve se certificar de que o conhecimento e as atitudes do paciente são corretos e completos.

Finalizando a dispensação o farmacêutico avalia a integridade física da embalagem para verificar se há alterações visíveis e também prazo de validade, devendo este ser compatível com o esquema de tratamento. Nesse momento podese reforçar as orientações prestadas e esclarecer alguma dúvida. Recomenda-se que a farmácia elabore material educativo impresso sobre o uso de medicamentos.

\section{Gestão da prática}

Para implementar esse modelo de dispensação é necessário disponibilizar equipe de pessoas capacitadas, infraestrutura adequada e procedimentos descritos e integrados à rotina da farmácia. 
O farmacêutico deve estar disponível para o atendimento de pacientes no balcão. Para isso não poderá acumular outras funções como a gerência da farmácia ou drogaria. Se não houver outro farmacêutico na empresa, essas atividades administrativas, inclusive o registro da movimentação de medicamentos controlados, deverão ser delegadas para outros profissionais administrativos.

O farmacêutico responsável pela dispensação deve ter conhecimento técnico, habilidade de comunicação com pacientes e outros profissionais de saúde, liderança para coordenar a equipe de auxiliares, e ainda, saber acolher, ouvir e ser empático. Essas características devem ser atualizadas e recicladas por meio de educação continuada.

Os atendentes devem ser treinados para auxiliar a dispensação, acolhendo o paciente, iniciando o processo e encaminhando aqueles que necessitam de atendimento farmacêutico.

A dispensação como está sendo proposta pode ser realizada no balcão. Porém, sugere-se que no local tenham cadeiras para favorecer o conforto do paciente/cliente e divisórias para propiciar privacidade no atendimento.

Como suporte para essa prática é interessante que a farmácia ofereça serviço de aferição de parâmetros físicos (pressão arterial, peso, altura, temperatura, glicemia capilar). Esses procedimentos ainda não estão totalmente regulamentados pela ANVISA, mas há intenção de normatização conforme Consulta Pública n. 69/2007.

Além disso, o farmacêutico deve contar com bibliografia básica para consulta rápida no balcão e para estudos mais aprofundados e, se possível, acesso à internet.

O farmacêutico pode desenvolver fichas para consulta rápida com informações sobre uso correto, precauções, principais interações, reações adversas e parâmetros de monitorização. Essas podem ser utilizadas também pelos atendentes. Outro material que pode ser elaborado são cartilhas com informações sobre o medicamento para o paciente.

Os procedimentos e as informações de suporte devem estar descritos, entendidos e disponíveis para consulta de toda a equipe. Esse material deve ser permanentemente atualizado pelo farmacêutico e pela equipe frequentemente treinada.

Como toda prática profissional, é importante que seja feita a documentação da dispensação realizada. Essa é uma atividade ignorada e dis- tante da rotina atual de uma farmácia ou drogaria, porém, devem ser feitos esforços para elaborar fichas para realizar registros que possibilitem a avaliação do serviço e a rastreabilidade do paciente/medicamento.

\section{Considerações Finais}

Há o consenso entre os farmacêuticos de que é necessária uma mudança da prática farmacêutica em farmácias e drogarias. Muito se tem discutido sobre a atenção farmacêutica e pouco empenho tem sido feito para melhorar as práticas anteriores, como a dispensação, que possibilita o atendimento de maior número de pessoas podendo causar impacto superior na sociedade. Exemplo disso é o reduzido número de publicações sobre o assunto. Acredita-se que haja farmacêuticos trabalhando sob a perspectiva apresentada, mas sem sistematização e divulgação. Este trabalho, então, também tem o propósito de iniciar uma discussão sobre o assunto, estimular a publicação de trabalhos com resultados da prática e de auxiliar no ensino farmacêutico.

A dispensação pode ser implementada a partir do modelo proposto, pois além de conjugar com a realidade de trabalho daqueles farmacêuticos que são comprometidos com o bem-estar de sua comunidade não entra em conflito com a característica comercial dos estabelecimentos farmacêuticos.

Outra vantagem de se implementar um modelo de prática é facilitar o desenvolvimento de um trabalho de qualidade e, a partir disso, possibilitar o reconhecimento pela sociedade tornando-se essencial para ela. Assim, a própria comunidade passa a exigir a atuação desse profissional para atender suas necessidades, independente da obrigatoriedade legal.

Novos estudos são necessários para propor modelos de documentação para registro da dispensação. Essa documentação deve possibilitar a obtenção de indicadores para gestão da prática e fornecer dados para estudos de avaliação do impacto da dispensação na saúde das pessoas.

A partir desse modelo, o farmacêutico pode participar do cuidado aos usuários de medicamentos interferindo, principalmente, no uso adequado, e isto se reflete de forma positiva nos resultados da terapia medicamentosa. 


\section{Colaboradores}

D Angonesi e MUP Rennó participaram igualmente de todas as etapas da elaboração do artigo.

\section{Agradecimentos}

Os autores agradecem à Doutora Djenane Ramalho de Oliveira pela revisão.

\section{Referências}

1. Hepler CD, Strand LM. Oportunities and responsabilities in pharmaceutical care. Am J Hosp Pharm. 1990; 47:533-543.

2. Angonesi D. Atenção Farmacêutica: Fundamentação conceitual e crítica para um modelo brasileiro. [monografia] Belo Horizonte: Universidade Federal de Minas Gerais; 2005.

3. Organização Panamericana de Saúde (OPAS). Consenso Brasileiro de Atenção Farmacêutica: Proposta. Brasília: OPAS, MS; 2002.

4. Organización Pan-Americana de la Salud (OPAS)/ Organización Mundial de la Salud (OMS). El papel del farmacéutico en la atencion a salud. Informe de la reunión de la OMS, Tokio, Japon, 31 ago. al 3 sep. de 1993. Washington: PAHO; 1995.

5. Cipolle RJ, Strand LM, Morley PC. El ejercicio de la atención farmacéutica. Madrid: McGraw-Hill Interamericana; 2000.

6. Angonesi D. Dispensação farmacêutica: uma análise de diferentes conceitos e modelos. Cien Saude Colet 2008; 13(Supl.):629-640.

7. Brasil. Portaria MS no 3916 de 30 de outubro de 1998. Aprova a Política Nacional de Medicamentos. Diário Oficial da União 1998; 10 nov.

8. Brasil. Lei no 5.991, de 17 de Dezembro de 1973. Dispõe sobre o controle sanitário do comércio de drogas, medicamentos, insumos farmacêuticos e correlatos, e dá outras providências. Diário Oficial da República Federativa do Brasil 1973; 19 dez.

9. España. Madrid. Ministério de Sanidad y Consumo. Consenso sobre atención Farmacéutica. España: Ministério de Sanidad y Consumo; 2001.

10. Dupim JAA. Assistência Farmacêutica: um modelo de organização. Belo Horizonte: SEGRAC; 1999.

11. Marin N, Luiza VL, Osório-de-Castro CGS, Machado-dos-Santos S, organizadores. Assistência Farmacêutica para gerentes municipais. Rio de Janeiro: OPAS, OMS; 2003.

12. Arias TD. Glosario de medicamentos: desarrollo, evaluación Y uso terminos especializados para la evaluación de medicamentos. Washington: OPAS; 1999.

13. Brasil. Portaria no 344, de 12 de Maio de 1998. Aprova o Regulamento Técnico sobre substâncias e medicamentos sujeitos a controle especial. Diário Oficial da República Federativa do Brasil 1998; 12 mai. 